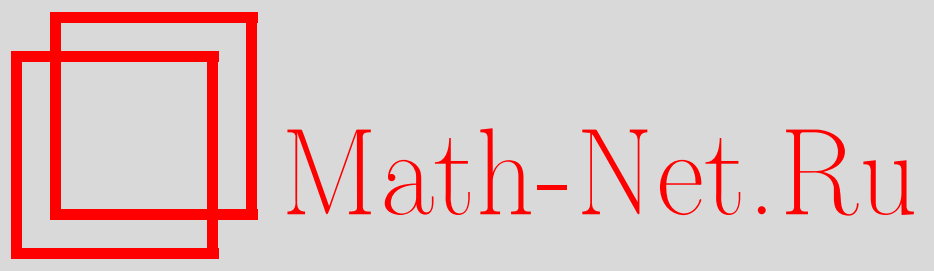

С. Ю. Катышев, В. Т. Марков, А. А. Нечаев, Использование неассоциативных группоидов для реализации процедуры открытого распределения ключей, Дискрет. матем., 2014, том 26, выпуск 3, 45-64

DOI: https://doi.org/10.4213/dm1289

Использование Общероссийского математического портала Math-Net.Ru подразумевает, что вы прочитали и согласны с пользовательским соглашением http: //www . mathnet.ru/rus/agreement

Параметры загрузки:

IP : 54.237 .206 .68

26 апреля 2023 г., 12:49:41

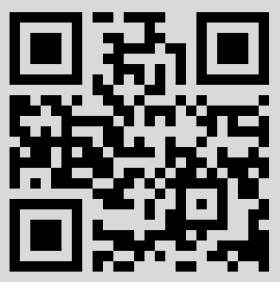




\section{Использование неассоциативных группоидов для реализации процедуры открытого распределения ключей}

() 2014 г. С. Ю. Катышев*, В. Т. Марков** , А. А. Нечаев ${ }^{\S}$

Изучается возможность использования неассоциативных группоидов для реализации процедуры открытого распределения ключей на основе алгоритма, обобщающего хорошо известный алгоритм Диффи-Хеллмана. Доказано существование неассоциативных группоидов, обладающих необходимым для этого свойством перестановочности степеней, и не являющихся группоидами с ассоциативными степенями.

Работа выполнена при поддержке гранта Президента РФ НШ-6260.2012.10 и Академии криптографии РФ.

Ключевые слова: открытое распределение ключей, алгоритм Диффи-Хеллмана, неассоциативные группоиды, медиальные квазигруппы, конечномерные алгебры.

\section{Введение}

Мы изучаем возможность обобщения хорошо известного алгоритма Диффи-Хеллмана [7], использующего циклические группы для реализации протокола открытого распределения ключей, на случай, когда вместо группы используется неассоциативный группоид. Введём соответствующие определения.

Для элемента $g$ конечного группоида $(\Omega, *)$ и заданных $r, l \in \mathbb{N}$ определим правую $r$-ю и левую l-ю степени соответственно равенствами:

$$
g^{[r]}=\underbrace{(\ldots((g * g) * g) \ldots)}_{\text {г сомножителей }}, \quad{ }^{[l]} g=\underbrace{(\ldots(g *(g * g)) \ldots)}_{\text {l сомножителей }} .
$$

Назовем $g$ элементом $c$ перестановочными правыми степенями или ППС-элементом, если

$$
\forall m, n \in \mathbb{N}: \quad g^{[m][n]}=g^{[n][m]} .
$$

* Место работы: ООО "Центр сертификационных исследований e-mail: sairos87@mail.ru

** Место работы: МГУ имени М.В. Ломоносова, e-mail: vtmarkov@yandex.ru

§ Место работы: Академия криптографии Российской Федерации, e-mail: alexnechaev@inbox.ru 
Если это тождество выполняется для всех элементов $g \in \Omega$, то будем называть $(\Omega, *)$ ППС-группоидом. Аналогично, с использованием тождества

$$
\forall m, n \in \mathbb{N}: \quad{ }^{[m][n]} g={ }^{[n][m]} g,
$$

определяются элементы и группоиды с перестановочными левыми степенями, ПЛС-элементы и ПЛС-группоиды, соответственно.

Алгоритм №1 открытого распределения ключей. Выбрав (несекретный) ППС-элемент $g$ группоида $\Omega$, абоненты $A$ и $B$ независимо друг от друга выбирают произвольные числа $r_{A}, r_{B} \in \mathbb{N}$, соответственно, и обмениваются элементами $g^{\left[r_{A}\right]}$ и $g^{\left[r_{B}\right]}$. Затем формируют общий секретный ключ $g^{\left[r_{A}\right]\left[r_{B}\right]}=g^{\left[r_{B}\right]\left[r_{A}\right]}$.

Сложность восстановления наблюдателем секретного ключа по открытой информации $g, g^{\left[r_{A}\right]}, g^{\left[r_{B}\right]}$ не превосходит сложности задачи правого дискретного логарифмирования в группоиде, т. е. сложности решения уравнения

$$
g^{[x]}=h .
$$

Естественным обобщением данного подхода к построению процедур открытого распределения ключей является комбинирование правых и левых степеней.

Будем говорить, что группоид $(\Omega, *)$ есть группоид с перестановочными степенями (ПС-группоид), если он является ПЛС- и ППС-группоидом, а также для любого $g \in \Omega$ и любых $l, r \in \mathbb{N}$ выполняется равенство

$$
{ }^{[l]}\left(g^{[r]}\right)=\left({ }^{[l]} g\right)^{[r]} \text {. }
$$

Будем говорить, что элемент $g$ произвольного группоида $(\Omega, *)$ есть $\Pi C$-элемент, если он порождает ПС-группоид.

С использованием ПС-элемента $g$ можно предложить

Алгоритм №2 открытого распределения ключей. Абонент А выбирает пару чисел

$$
\left(l_{A}, r_{A}\right), \quad l_{A}, r_{A} \in \mathbb{N}
$$

а абонент В - пару

$$
\left(l_{B}, r_{B}\right), \quad l_{B}, r_{B} \in \mathbb{N} .
$$

Абоненты А и В открыто обмениваются элементами ${ }^{\left[l_{A}\right]} g^{\left[r_{A}\right]}$ и ${ }^{\left[l_{B}\right]} g^{\left[r_{B}\right]}$. В качестве общего ключа выбирается элемент

$$
{ }^{\left[l_{A}\right]}\left({ }^{\left[l_{B}\right]} g^{\left[r_{B}\right]}\right)^{\left[r_{A}\right]}={ }^{\left[l_{B}\right]}\left({ }^{\left[l_{A}\right]} g^{\left[r_{A}\right]}\right)^{\left[r_{B}\right]} .
$$

При такой процедуре сложность вычисления общего секретного ключа наблюдателем оценивается сверху сложностью решения задачи лево-правого логарифмирования, т. е. сложностью решения уравнения с двумя неизвестными $x, y$ :

$$
{ }^{[x]} g^{[y]}=h .
$$

Естественным обобщением последнего алгоритма является

Алгоритм №3 открытого распределения ключей. Абоненты $A$ и $B$ независимо друг от друга выбирают натуральные числа $r, m, s, n$ такие, что $r \leqslant m, s \leqslant n$ и наборы чисел, соответственно,

$$
a_{1}, \ldots, a_{m} \in \mathbb{N} ; \quad \text { и } \quad b_{1}, \ldots, b_{n} \in \mathbb{N}
$$


обмениваются сообщениями

$$
K_{A}={ }^{\left[a_{1}\right] \ldots\left[a_{r}\right]} g^{\left[a_{r+1}\right] \ldots\left[a_{m}\right]}, \quad \text { и } K_{B}={ }^{\left[b_{1}\right] \ldots\left[b_{s}\right]} g^{\left[b_{s+1}\right] \ldots\left[b_{n}\right]} .
$$

Абоненты $A$ и $B$ вырабатывают общий ключ $K_{A B}$ :

$$
{ }^{\left[a_{1}\right] \ldots\left[a_{r}\right]} K_{B}^{\left[a_{r+1}\right] \ldots\left[a_{m}\right]}={ }^{\left[b_{1}\right] \ldots\left[b_{s}\right]} K_{A}^{\left[b_{s+1}\right] \ldots\left[b_{n}\right]} .
$$

В разделе 2.2 будет показано, что нахождение общего секретного ключа в алгоритме 3 не сложнее решения задачи обобщенного дискретного логарифмирования, т.е, нахождения хотя бы одной пары натуральных чисел $u, v$ и набора $\left(x_{1}, \ldots, x_{v}\right)$ $x_{i} \in \mathbb{N}, i \in \overline{1, v}$, удовлетворяющих равенству

$$
\left[x_{1}\right] \ldots\left[x_{u}\right] g^{\left[x_{u+1}\right] \ldots\left[x_{v}\right]}=h,
$$

если такие существуют.

Сложность решения уравнений (2), (3), (5) определяют, прежде всего, два фактора:

(i) выбор алгебраического носителя алгоритма: группоида $(\Omega, *)$ и элемента $g$, обеспечивающий стойкость алгоритма по отношению к методам, связанным с факторизацией группоида и перебором;

(ii) выбор способа представления алгебраического носителя, при котором функция возведения в правую (левую) степень однонаправленная.

В качестве верхней оценки стойкости алгоритма Диффи-Хеллмана, с учетом этих двух факторов, принято выбирать сложность логарифмирования на произвольной циклической группе порядка $n$. Будем обозначать сложность такого логарифмирования через $L g(n)$. Известна следующая общая оценка этой функции: $L g(n)=O(\sqrt{n})$ (сложность логарифмирования методом согласования [7, 2]). В настоящее время эта оценка понижена для ряда значений числа $n$ и способов представления абелевой группы до субэкспоненциальной (от размера задачи $\log _{2} n$ ) оценки. Однако она остается лучшей в классе детерминированных алгоритмов для случая, когда $n-$ простое и $n-1=2 p, \quad p$ - простое.

Следует отметить, что последняя оценка является классическим примером экспертной оценки, т. е. не доказано то, что она является нижней, но многочисленные усилия ученых не привели к её уменьшению способом, не зависящим от способа представления алгебраического носителя (см. (ii)).

В данной статье, прежде всего, доказывается существование нужных для алгоритмов 1-3 алгебраических носителей: ПС-группоидов с неассоциативными степенями $\left(g^{[m][n]} \neq g^{[m n]}\right)$. Дается описание некоторых классов таких группоидов, интересных для дальнейшего изучения с точки зрения возможности реализации достаточно стойкого алгоритма открытого распределения ключей.

\section{1. Построение классов ПС-группоидов}

1.1. Числовые характеристики элемента конечного группоида Пусть $(\Omega, *)$ - произвольный конечный группоид. Для произвольного элемента $g \in \Omega$ обозначим через $g^{\mathbb{N}}=\left\{g^{[n]}\right\}_{n \in \mathbb{N}}$ последовательность его степеней.

Предложение 1. Последовательность $g^{\mathbb{N}}$ периодическая. 
Доказательство. Для любых $a_{1}, \ldots, a_{n} \in \Omega$ введём обозначение

$$
\rho_{n}\left(a_{1}, \ldots, a_{n}\right)=\left(\ldots\left(\left(a_{1} * a_{2}\right) * a_{3}\right) \ldots\right) * a_{n} .
$$

Заметим, что при этом $a_{1} * a_{2}=\rho_{2}\left(a_{1}, a_{2}\right)$ и, в частности, если $a \in \Omega, \quad 1<k<n$, то

$$
a^{[n]}=\rho_{n}(a, \ldots a, \ldots, a)=\rho_{n-k+1}\left(\rho_{k}(a, \ldots, a), a, \ldots, a\right)=\rho_{n-k+1}\left(a^{[k]}, a, \ldots, a\right) .
$$

Тогда для любых $n, k, x \in \mathbb{N}$ справедлива импликация:

$$
g^{[n]}=g^{[k]} \Rightarrow g^{[n+x]}=g^{[k+x]},
$$

так как $g^{[n+x]}=\rho_{x+1}\left(g^{[n]}, g, \ldots, g\right)=\rho_{x+1}\left(g^{[k]}, g, \ldots, g\right)=g^{[k+x]}$

Остается заметить, что в силу конечности группоида $(\Omega, *)$ в последовательности $g^{\mathbb{N}}$ встретятся повторяющиеся элементы.

Определим правый дефект $D_{r}(g)$ и правый период $T_{r}(g)$ элемента $g$ соответственно как дефект и период последовательности $g^{\mathbb{N}}$ :

$$
\begin{aligned}
& D_{r}(g)=\min \left\{l \in \mathbb{N}_{0}: \quad \exists t \in \mathbb{N} \forall i \in \mathbb{N} \quad g^{[l+i+t]}=g^{[l+i]}\right\}, \\
& T_{r}(g)=\min \left\{t \in \mathbb{N}: \quad \forall i \in \mathbb{N} \quad g^{\left[D_{r}(g)+i+t\right]}=g^{\left[D_{r}(g)+i\right]}\right\} .
\end{aligned}
$$

Аналогично определим левые дефект $-D_{l}(g)$ и период $-T_{l}(g)$.

Хорошо известные свойства периодических последовательностей (см., например, [5]) обосновывают следующее утверждение.

Предложение 2. Пусть $g$ - элемент произвольного конечного группоида $(\Omega, *)$. Тогда:

1) Eсли $t, t^{\prime} \in \mathbb{N}, u \quad t^{\prime}>t, m o$

$$
g^{[t]}=g^{\left[t^{\prime}\right]} \Leftrightarrow t \geqslant D_{r}(g), T_{r}(g) \mid t^{\prime}-t .
$$

2) Справедливо неравенство $D_{r}(g)+T_{r}(g) \leqslant|\Omega| \quad\left(D_{l}(g)+T_{l}(g) \leqslant|\Omega|\right)$.

Определим правыци потенциал (левый потенциал) произвольного элемента $g \in \Omega$ как $t \in \mathbb{N}$ такое наибольшее, что все элементы $g, g^{[2]}, \ldots, g^{[t]} \quad\left(g,{ }^{[2]} g, \ldots,{ }^{[t]} g\right)$ различны. Обозначим эту величину $\operatorname{pot}_{r} g\left(\operatorname{pot}_{l} g\right)$, или $\operatorname{pot}_{r *} g\left(\operatorname{pot}_{l^{*}} g\right)$, когда необходимо подчеркнуть зависимость от операции $*$.

Справедливы равенства

$$
D_{r}(g)+T_{r}(g)=\operatorname{pot}_{r}(g), \quad D_{l}(g)+T_{l}(g)=\operatorname{pot}_{l}(g) .
$$

Будем называть элемент $g \in \Omega$ реверсивным $\left(\right.$ справа), если $D_{r}(g)=0$, т. е. $g^{[t+1]}=g$ для некоторого $t \in \mathbb{N}$, или, другими словами, $T_{r}(g)=\operatorname{pot}_{r}(g)$.

Из предложения 2 видно, что функция "потенциал" для реверсивных элементов группоида обладает свойством, аналогичным основному свойству функции "порядок" для элементов группы.

Предложение 3. Пусть $g$ - реверсивный элемент группоида $(\Omega, *)$. Тогда

$$
\forall t, t^{\prime} \in \mathbb{N}: \quad g^{[t]}=g^{\left[t^{\prime}\right]} \Longleftrightarrow \operatorname{pot}_{r} g \mid t^{\prime}-t .
$$

Следствие 1. Если $(\Omega, *)$ - квазигруппа с единищей е (лупа), то

$$
\forall g \in \Omega, \quad t \in \mathbb{N}_{0} \quad g^{t}=e \Longleftrightarrow \operatorname{pot}_{r} g \mid t .
$$


В определении перестановочности степеней элемента $g$ группоида $(\Omega, *)$ формально используется бесконечное число тождеств. Покажем, что для проверки того, что $g$ - ПС-элемент, достаточно проверить конечное число тождеств.

Определим правый дефект $D_{r}=D_{r}(\Omega)$ группоида $(\Omega, *)$ и его правый период $T_{r}$ соотношениями

$$
D_{r}=\max \left\{D_{r}(g), g \in \Omega\right\}, \quad T_{r}=H O K\left\{T_{r}(g), g \in \Omega\right\} .
$$

Для каждого элемента $g$ группоида выполнены соотношения

$$
\forall i>D_{r}: g^{[i]}=g^{\left[i+T_{r}\right]} .
$$

Предложение 4. Элемент g группоида $(\Omega, *)$ является ППС-элементом тогда и только тогда, когда

$$
\forall n, k \in \overline{1, D_{r}+T_{r}}: \quad\left(g^{[n]}\right)^{[k]}=\left(g^{[k]}\right)^{[n]} .
$$

Доказательство. Необходимость (9) для (1) очевидна. Покажем достаточность. Для $n \in \mathbb{N}$ определим значение $\rho(n)$ условиями

$$
\rho(n)=\left\{\begin{array}{l}
n, \text { если } n \leqslant D_{r}+T_{r} ; \\
n-k T_{r}, \text { где } k \in \mathbb{N}_{0} \quad \text { и }\left(n-k T_{r}\right) \in \overline{D_{r}, D_{r}+T_{r}}, \text { если } n>D_{r}+T_{r} .
\end{array}\right.
$$

Очевидно, для любых $g \in \Omega, n \in \mathbb{N}$ выполнено равенство $g^{[n]}=g^{[\rho(n)]}$. Следовательно для любых $n, k \in \mathbb{N}$ верна цепочка равенств

$$
\left(g^{[n]}\right)^{[k]}=\left(g^{[\rho(n)]}\right)^{[k]}=\left(g^{[\rho(n)]}\right)^{[\rho(k)]}=\left(g^{[\rho(k)]}\right)^{[\rho(n)]}=\left(g^{[k]}\right)^{[\rho(n)]}=\left(g^{[k]}\right)^{[n]} .
$$

Аналогично формулируются критерии перестановочности левых и лево-правых степеней.

В следующих параграфах покажем, что существуют широкие классы ПС-группоидов (возможных алгебраических носителей процедуры открытого формирования ключа) произвольно большой мощности.

1.2. Локально-медиальные группоиды В данном параграфе обобщены частные неопубликованные результаты А. Н. Дмитриевской, Е. Н. Дмитриевской, Ф. К. Зоркого.

Пусть $(\Omega, \cdot)$ - произвольная конечная полугруппа с единицей $e$. Всюду далее будем считать, что $g^{0}=e$ для любого $g \in \Omega$. Пусть $\left(\Omega^{\Omega}\right.$, о $)-$ полугруппа всех отображений $\Omega \rightarrow \Omega$ и $\widetilde{\operatorname{Aut}}(\Omega)=\widetilde{\operatorname{Aut}}(\Omega, \cdot)-$ подгруппа полугруппы $\left(\Omega^{\Omega}, \circ\right)$, состоящая из всех автоморфизмов и антиавтоморфизмов полугруппы $(\Omega, \cdot)$.

Зафиксируем два коммутирующих (анти-)автоморфизма

$$
\sigma, \tau \in \widetilde{\operatorname{Aut}}(\Omega), \quad \sigma \tau=\tau \sigma,
$$

и зададим на $\Omega$ новую операцию $*$ условием:

$$
\forall x, y \in \Omega \quad x * y=\sigma(x) \cdot \tau(y) .
$$

Мы получим группоид $(\Omega, *)$, который является квазигруппой тогда и только тогда, когда $(\Omega, \cdot)$ - группа. Такие квазигруппы относят к классу медиальных квазигpynn [3].

Тогда по аналоги будем называть группоид $(\Omega, *)$ медиальнъм, если $(\Omega, \cdot)$ - абелева полугруппа. 
Теорема 1. Если $(\Omega, \cdot)$ - абелева полугруппа и $\sigma, \tau-$ ее коммутирующие (анти-)автоморбизмы, то группоид $(\Omega, *)$ с операцией (11) есть ПС-группоид.

Доказательство. Нетрудно увидеть, что для $g \in(\Omega, *), m \in \mathbb{N}$ выполнены равенства

$$
\begin{aligned}
& g^{[m]}=\sigma^{m-1}(g) \cdot \sigma^{m-2} \tau(g) \cdot \ldots \cdot \sigma \tau(g) \cdot \tau(g)=\sigma^{m-1}(g) \prod_{i=0}^{m-2} \sigma^{i} \tau(g), \\
& { }^{[m]} g=\sigma(g) \cdot \sigma \tau(g) \cdot \ldots \cdot \sigma \tau^{m-2}(g) \cdot \tau^{m-1}(g)=\tau^{m-1}(g) \prod_{i=0}^{m-2} \tau^{i} \sigma(g) .
\end{aligned}
$$

Пользуясь (12), получаем, что для любых $k, m \in \mathbb{N}$

$$
\left(g^{[k]}\right)^{[m]}=\sigma^{m-1}\left(g^{[k]}\right) \prod_{i=0}^{m-2} \sigma^{i}\left(\tau\left(g^{[k]}\right)\right) .
$$

Подставляя сюда вместо $g^{[k]}$ правую часть (12) при $m=k$, пользуясь коммутативностью полугруппы $(\Omega, \cdot)$ и перестановочностью автоморфизмов $(10)$, получаем:

$$
\begin{aligned}
\left(g^{[k]}\right)^{[m]}=\sigma^{m+k-2}(g) \prod_{j=0}^{k-2} \sigma^{m-1+j}(\tau(g)) & \prod_{i=0}^{m-2} \sigma^{k-1+i}(\tau(g)) \\
& \prod_{i=0}^{m-2} \prod_{j=0}^{k-2} \sigma^{i+j}\left(\tau^{2}(g)\right) .
\end{aligned}
$$

Нетрудно заметить, что правая часть полученного выражения симметрична относительно $k$ и $m$. Действительно, если заменить $m$ на $k$ и $k$ на $m$, то первый сомножитель и произведение во второй строке в (15) не изменятся, а средние два произведения поменяются местами. Это не изменит значение в правой части, так как полугруппа $(\Omega, \cdot)$ абелева.

Аналогично, используя (12), (13), доказывается перестановочность левых степеней элемента $g$.

Теперь покажем, что для любых $l, r \in \mathbb{N}, g \in \Omega$ выполняется равенство

$$
{ }^{[l]}\left(g^{[r]}\right)=\left({ }^{[l]} g\right)^{[r]} \text {. }
$$

Из (13) получаем:

$$
{ }^{[l]}\left(g^{[r]}\right)=\tau^{l-1}\left(g^{[r]}\right) \prod_{i=0}^{l-2} \tau^{i} \sigma\left(g^{[r]}\right) .
$$

Отсюда, пользуясь (12) и коммутативностью полугруппы $(\Omega, \cdot)$, имеем:

$$
\begin{aligned}
{ }^{[l]}\left(g^{[r]}\right)= & \tau^{l-1}\left(\sigma^{r-1}(g) \prod_{j=0}^{r-2} \sigma^{j} \tau(g)\right) \prod_{i=0}^{l-2} \tau^{i} \sigma\left(\sigma^{r-1}(g) \prod_{j=0}^{r-2} \sigma^{j} \tau(g)\right)= \\
& \left.\left.\left(\tau^{l-1} \sigma^{r-1}(g)\right)\left(\prod_{j=0}^{r-2} \sigma^{j} \tau^{l}(g)\right)\right)\left(\prod_{i=0}^{l-2} \tau^{i} \sigma^{r}(g)\right)\left(\prod_{i=0}^{l-2} \prod_{j=0}^{r-2} \sigma^{j+1} \tau^{i+1}(g)\right)\right) .
\end{aligned}
$$

Из (12), (13) легко следует, что соответствующее равенство для $\left({ }^{[l]} g\right)^{[r]}$ получается из правой части (17) перестановкой $\sigma$ и $\tau, l$ и $r$. 
Ввиду перестановочности автоморфизмов $\sigma$ и $\tau$ после этих преобразований выражение в правой части равенства (17) не изменяется. Таким образом, $(\Omega, *)-$ ПС-группоид.

Локально-медиальные группоиды. Покажем, что условия коммутативности полугруппы в теореме 1 можно несколько ослабить. Пусть $(\Omega, \cdot)$ - произвольная полугруппа, выполнено условие (10) и $H=\langle\sigma, \tau\rangle$ - подгруппа группы $\widehat{\operatorname{Aut}}(\Omega)$, порожденная автоморфизмами $\sigma, \tau$. Тогда для группоида $(\Omega, *)$ с операцией $(11)$ справедлива следующая теорема.

Теорема 2. Если для некоторого элемента $g \in \Omega$ элементы орбиты $H(g)$ попарно перестановочны в $(\Omega, \cdot)$, то g есть ПС-элемент. Если последнее условие перестановочности выполняется для всех $g \in \Omega$, то $(\Omega, *)$ есть ПC-группоид.

Доказательство. Заметим, что подгруппоид, порожденный элементом $g$ в группоиде $(\Omega, *)$, содержится в коммутативной подполугруппе $S$ полугруппы $(\Omega, \cdot)$, порожденной множеством $H(g)$. Эта подполугруппа является также подгруппоидом в группоиде $(\Omega, *)$, и к ней можно применить теорему 5 , рассматривая вместо $\sigma$ и $\tau$ их ограничения на подполугруппу $S$.

Будем называть группоиды из последней теоремы локально-медиальным на элементе g и локально-медиальным, соответственно.

Пример 1. Пусть $(\Omega, \cdot)$-произвольная конечная группа, $\sigma=\mathrm{id}_{\Omega}-$ тождественный автоморфизм и $\tau-$ (анти)автоморфизм, вида $\tau(g)=g^{-1}$. Тогда группоид $(\Omega, *)$ с операцией (11) является локально-медиальным.

Дополнительно докажем следующее свойство потенциалов элементов локально-медиального группоида, построенного на группе.

Предложение 5. Пусть $(\Omega, \cdot)$ группа. Если $(\Omega, *)$ - локально-медиальный группоид с операчией (11), то для любого $g \in \Omega$, справедливо соотношение

$$
\operatorname{pot}_{r} g \mid \operatorname{ord} \sigma \cdot \operatorname{ord} g \text {. }
$$

Доказательство. Пусть ord $\sigma=s$, ord $g=t$. Тогда $\sigma^{s}=\varepsilon-$ тождественный автоморфизм и $g^{t}=e-$ единица группы $(\Omega, \cdot)$. Используя $(12)$, получаем:

$$
g^{[s t+1]}=\sigma^{s t}(g) \cdot \sigma^{s t-1}(\tau(g)) \cdot \sigma^{s t-2}(\tau(g)) \cdot \ldots \cdot \sigma(\tau(g)) \cdot \tau(g) .
$$

Отсюда, обозначая $h=\sigma^{s-1}(\tau(g)) \cdot \ldots \cdot \sigma(\tau(g)) \cdot \tau(g)$ и пользуясь равенством $\sigma^{s}(h)=h$, приходим к равенствам $g^{[s t+1]}=\sigma^{s t}(g) \cdot h^{t}=\varepsilon^{t}(g) \cdot h^{t}=g \cdot h^{t}$. Так как рассматриваемый группоид локально-медиальный, то любые два элемента вида $\sigma^{i}(\tau(g)), \sigma^{j}(\tau(g))$ перестановочны и

$$
\begin{array}{r}
h^{t}=\sigma^{s-1}(\tau(g))^{t} \cdot \ldots \cdot \sigma(\tau(g))^{t} \cdot \tau(g)^{t}=\sigma^{s-1}\left(\tau\left(g^{t}\right)\right) \cdot \ldots \cdot \sigma\left(\tau\left(g^{t}\right)\right) \cdot \tau\left(g^{t}\right)= \\
=\sigma^{s-1}(\tau(e)) \cdot \ldots \cdot \sigma(\tau(e)) \cdot \tau(e)=e .
\end{array}
$$

Таким образом, $g^{[s t+1]}=g$. Остается использовать предложение 3 . 


\section{3. Оценка сложности вычисления степени элемента в} локально-медиальном группоиде Из равенства (12) следует, что в группоиде с операцией (11) при вычислении правых степеней правый автоморфизм $\tau$ ведет себя "весьма пассивно". В связи с этим возникает вопрос: не будет ли этот группоид изоморфен некоторому группоиду $(\Omega, \star)$ с операцией

$$
\forall x, y \in \Omega: \quad x \star y=\varphi(x) y, \quad \varphi \in \operatorname{Aut}(\Omega) ?
$$

Предложение 6. Если $(\Omega, \cdot)$ - полугруппа с единищей е и операции (11), (18) таковы, что $(\Omega, *) \cong(\Omega, \star)$, то $\tau-$ тождественный автоморфизм.

Доказательство. Нетрудно проверить, что элемент $e$ - единственная левая единица в группоиде $(\Omega, \star)$, а группоид $(\Omega, *)$ имеет левую единицу тогда и только тогда, когда $\tau=\varepsilon$.

Если $(\Omega, *)$ - локально-медиальный группоид, то даже при $\tau \neq \varepsilon$ задачи о вычислении степеней и логарифмировании в этом группоиде можно свести к решению аналогичных задач в группоиде с операцией вида $(18)$. Пусть $(\Omega, \star)$ - группоид с операцией

$$
\forall x, y \in \Omega: \quad x \star y=\sigma(x) \cdot y .
$$

Заметим, что если $(\Omega, *)$ - локально-медиальный группоид, то таков же и группоид $(\Omega, \star)$.

Для произвольного $\alpha \in \Omega$ обозначим через $\alpha^{[* n]}$ и $\alpha^{[\star n]}$ правые $n$-е степени элемента $\alpha$ соответственно в $(\Omega, *)$ и в $(\Omega, \star)$. Заметим, что

$$
\alpha^{[\star n]}=\sigma^{n-1}(\alpha) \cdot \ldots \cdot \sigma(\alpha) \cdot \alpha .
$$

Предложение 7. Пусть $(\Omega, \cdot)$ - полугруппа с единицей е. Если $g$ - обратимый элемент полугруппы $(\Omega, \cdot)$ и группоид $(\Omega, *)$ - локально-медиальный на элементе $g$ с операцией (11), то для операции (19) при любом $n \in \mathbb{N}$ справедливо равенство

$$
g^{[* n]}=g \cdot \alpha^{[\star n-1]},
$$

где $\alpha=\sigma(g) \tau(g) g^{-1}$. B частности, pot $_{r *} g=$ pot $_{r \star} \alpha$.

Доказательство. При $n=2$, пользуясь перестановочностью в $(\Omega, \cdot)$ элемента $g$ с элементами $\sigma(g), \tau(g)$, имеем:

$$
g^{[2]}=\sigma(g) \tau(g)=\sigma(g) \tau(g) g g^{-1}=g \cdot \sigma(g) \tau(g) g^{-1}=g \cdot \alpha .
$$

Пусть утверждение верно для $n=k \geqslant 2$. Покажем что оно верно для $n=k+1$. По определению $g^{[* k+1]}=g^{[* k]} * g=\sigma\left(g^{[* k]}\right) \tau(g)$. Отсюда, пользуясь предположением индукции, получаем: $g^{[* k+1]}=\sigma\left(g^{[* k]}\right) \tau(g)=\sigma\left(g \alpha^{[\star k-1]}\right) \tau(g)$.

Лемма 1. В условиях предложения 7 справедливы равенства

$$
g \alpha^{[\star k-1]}=\alpha^{[\star k-1]} g, \quad g \sigma\left(\alpha^{[\star k-1]}\right)=\sigma\left(\alpha^{[\star k-1]}\right) g .
$$

Доказательство. Ввиду (20) достаточно доказать, что для любого $i \in \mathbb{N}_{0}$ элемент $g$ коммутирует с элементом $\sigma^{i}(\alpha)=\sigma^{i+1}(g) \sigma^{i}(\tau(g)) \sigma^{i}\left(g^{-1}\right)$. Перестановочность $g$ с первыми двумя сомножителями в этом произведении следует из локальной медиальности группоида $(\Omega, *)$ на элементе $g$. Остается заметить, что так как $(\Omega, \cdot)$ - конечная полугруппа, то $g^{-1}=g^{t}$ для некоторого $t \in \mathbb{N}$, поэтому $g \sigma^{i}\left(g^{-1}\right)=g \sigma^{i}\left(g^{t}\right)=g\left(\sigma^{i}(g)\right)^{t}=\left(\sigma^{i}(g)\right)^{t} g=\sigma^{i}\left(g^{-1}\right) g$. 
С использованием леммы 1 доказательство предложения 7 завершается следующим образом:

$$
\begin{gathered}
g^{[* k+1]}=\sigma\left(g \alpha^{[\star k-1]}\right) \tau(g)=\sigma\left(\alpha^{[\star k-1]}\right) \sigma(g) \tau(g)=\sigma\left(\alpha^{[\star k-1]}\right) \sigma(g) \tau(g) g g^{-1}= \\
=g \sigma\left(\alpha^{[\star k-1]}\right) \sigma(g) \tau(g) g^{-1}=g \sigma\left(\alpha^{[\star k-1]}\right) \alpha=g\left(\left(\alpha^{[\star k-1]}\right) \star \alpha\right)=g \alpha^{[\star k]} .
\end{gathered}
$$

Теперь докажем ряд вспомогательных утверждений.

Предложение 8. Если $(\Omega, *)$ - локально-медиальный группоид с операщией (11) $u$ $\sigma, \tau \in \operatorname{Aut}(\Omega, \cdot)$, то для любых $a_{1}, a_{2}, \ldots, a_{n} \in \Omega, n>2$ в обозначениях (6) справедливо равенство

$$
\rho_{n}\left(a_{1}, \ldots, a_{n}\right)=\sigma^{n-2}\left(a_{1}\right) * \rho_{n-1}\left(a_{2}, \tau^{-1}\left(a_{3}\right), \ldots, \tau^{-1}\left(a_{n}\right)\right)
$$

Доказательство. Индукция по $n \geqslant 3$. Для $n=3$ имеем:

$$
\begin{aligned}
& \rho_{3}\left(a_{1}, a_{2}, a_{3}\right)=\left(a_{1} * a_{2}\right) * a_{3}=\sigma\left(\sigma\left(a_{1}\right) \cdot \tau\left(a_{2}\right)\right) \cdot \tau\left(a_{3}\right)= \\
= & \sigma\left(\sigma\left(a_{1}\right)\right) \cdot \tau\left(\sigma\left(a_{2}\right) \cdot \tau\left(\tau^{-1}\left(a_{3}\right)\right)\right)=\sigma\left(a_{1}\right) *\left(a_{2} * \tau^{-1}\left(a_{3}\right)\right) .
\end{aligned}
$$

Предположим, что утверждение верно для $n=k \geqslant 3$, то есть справедливо равенство

$$
\rho_{k}\left(a_{1}, \ldots, a_{k}\right)=\sigma^{k-2}\left(a_{1}\right) * \rho_{k-1}\left(a_{2}, \tau^{-1}\left(a_{3}\right), \ldots, \tau^{-1}\left(a_{k}\right)\right) .
$$

Для доказательства шага индукции умножим обе части этого равенства на $a_{k+1}$ справа и еще раз воспользуемся предположением индукции:

$$
\begin{gathered}
\rho_{k+1}\left(a_{1}, \ldots, a_{k}, a_{k+1}\right)=\rho_{k}\left(a_{1}, \ldots, a_{k}\right) * a_{k+1}= \\
=\left(\sigma^{k-2}\left(a_{1}\right) * \rho_{k-1}\left(a_{2}, \tau^{-1}\left(a_{3}\right), \ldots, \tau^{-1}\left(a_{k}\right)\right)\right) * a_{k+1}= \\
=\rho_{3}\left(\sigma^{k-2}\left(a_{1}\right), \rho_{k-1}\left(a_{2}, \tau^{-1}\left(a_{3}\right), \ldots, \tau^{-1}\left(a_{k}\right)\right), a_{k+1}\right)= \\
=\sigma\left(\sigma^{k-2}\left(a_{1}\right)\right) * \rho_{2}\left(\rho\left(a_{2}, \tau^{-1}\left(a_{3}\right), \ldots, \tau^{-1}\left(a_{k}\right)\right), \tau^{-1}\left(a_{k+1}\right)\right)= \\
=\sigma^{(k+1)-2}\left(a_{1}\right) * \rho_{k}\left(a_{2}, \tau^{-1}\left(a_{3}\right), \ldots, \tau^{-1}\left(a_{k+1}\right) .\right.
\end{gathered}
$$

Предложение 9. Пусть $(\Omega, *)$ - локально-медиальный группоид, определенньй соотношениями (10), (11), и $a_{1}, a_{2}, \ldots, a_{n}-$ произвольные элементы $\Omega$. Тогда, если элементы множества $\mathcal{H}=H\left(a_{1}\right) \cup H\left(a_{2}\right) \cup \ldots \cup H\left(a_{n}\right)$ попарно перестановочны, то для элементов $a_{1}, a_{2}, \ldots, a_{n}$ справедливо равенство (22).

Доказательство. Если $\sigma, \tau$ - автоморфизмы, то требуемое равенство непосредственно следует из предложения 8. Если, например, $\sigma$ антиавтоморфизм, то его действие на элементах множества $\mathcal{H}$ в локально-медиальном группоиде $(\Omega, *)$ выглядит следующим образом:

$$
\forall h_{1}, h_{2} \in \mathcal{H} \quad \sigma\left(h_{1} \cdot h_{2}\right)=\sigma\left(h_{2}\right) \cdot \sigma\left(h_{1}\right)=\sigma\left(h_{1}\right) \cdot \sigma\left(h_{2}\right) .
$$

А значит, на элементах множества $\mathcal{H}$ антиавтоморфизм $\sigma$ ведет себя как автоморфизм. Поэтому для элементов $a_{1}, a_{2}, \ldots, a_{n}$ доказательство равенства (22) дословно повторяет доказательство предложения 8.

Следствие 2. Если $(\Omega, \star)$ - локально-медиальный группоид с операцией (19), то для любых $g \in \Omega, n, k \in \mathbb{N}$ справедливо равенство $g^{[\star n+k]}=\sigma^{k}\left(g^{[\star n]}\right) \cdot g^{[\star k]}$. 
Доказательство. Операция ^ представляется в виде (11) при $\tau=\varepsilon$. Тогда $\tau^{-1}=\varepsilon$ и, применяя формулы (7) и (22), получаем:

$$
\begin{gathered}
g^{[\star n+k]}=\rho_{n+k}(g, \ldots, g)=\rho_{k+1}\left(g^{[\star n]}, g, \ldots, g\right)= \\
\sigma^{k-1}\left(g^{[\star n]}\right) \star \rho_{k}(g, \ldots, g)=\sigma^{k}\left(g^{[\star n]}\right) \cdot g^{[k]} .
\end{gathered}
$$

Далее используются следующие обозначения. Пусть $\lfloor x\rfloor-$ наибольшее целое число, не превосходящее $x$. Для произвольных $h \in \Omega$ и $n, k \in \mathbb{N}$, где $n \leqslant 2^{k}$, введём обозначение

$$
h^{\{n / i\}}=\sigma^{\left\lfloor\frac{n}{2^{i}}\right\rfloor}(h) \cdot h .
$$

Заметим, что выражение в правой части (23) определено корректно, так как $\left\lfloor\frac{n}{2^{i}}\right\rfloor \in$ $\mathbb{N}$.

Сложность возведения в степень (анти-)автоморфизма, заданного на полугруппе, зависит от способа его задания. Обозначим через $A U T(\sigma, t)$ трудоёмкость вычисления значения $\sigma^{t}(h)$ для произвольного элемента $h$ полугруппы $(\Omega, \cdot)$.

Пусть элемент $g \in \Omega$ обратим в $(\Omega, \cdot)$. Для вычисления его степени в произвольном локально-медиальном группоиде предлагается следующий алгоритм.

\section{Алгоритм 3.}

Дано. Полугруппа $(\Omega, \cdot)$ с единицей $е,($ анти-) автоморфизмы $\sigma, \tau \in \widetilde{\operatorname{Aut}}(\Omega, \cdot)$, элемент $g \in \Omega$, обратимый в $(\Omega, \cdot), m \in \mathbb{N}$.

Выход. Элемент $g^{[* m]}$.

Шаг О. Если $m=1$, то вывести ответ $g$, иначе - иаг 1.

Шаг 1. Вычислить элемент $\alpha$ по формуле

$$
\alpha=\sigma(g) \tau(g) g^{-1}
$$

Шаг 2. Присвоить $h=\alpha^{n_{k}}, i=k$, где $k$ и $n_{k}$ - элементы двоичного разложения числа $n=m-1$ :

$$
n=\sum_{i=0}^{k} n_{i} 2^{i}, k=k(n)=\left\lfloor\log _{2} n\right\rfloor .
$$

Шаг 3. Пока $i>0$ : повторять:

1) Возвести автоморфизм $\sigma$ в степень $t=\left\lfloor\frac{n}{2^{i}}\right\rfloor: \delta=\sigma^{t}$;

2) Вычислить новое значение элемента $h: h=\sigma(\delta(h) \cdot h) \cdot \alpha^{n_{i-1}}$;

3) Присвоить: $i=i-1$.

Шаг 4. Вывести ответ $g^{[m]}=g h$.

Для доказательства корректности работы алгоритма докажем лемму.

Лемма 2. Если $(\Omega, \star)$ - локально медиалъньй группоид с операцией (19), то для любых $\alpha \in \Omega$ u $n \in \mathbb{N}$ с разложением (24) справедливо равенство

$$
\alpha^{[\star n]}=\left(\ldots\left(\left(\alpha^{n_{k}}\right)^{\{n / k\}} \star \alpha^{n_{k-1}}\right)^{\{n / k-1\}} \star \ldots \star \alpha^{n_{1}}\right)^{\{n / 1\}} \star \alpha^{n_{0}} .
$$

Доказательство. Доказательство проведем индукцией по параметру $k=k(n) \geqslant 0$ в разложении (24). Если $k=0$, то $n=n_{0}=1$ и равенство (25) очевидно.

Пусть $m>0$ и утверждение верно для всех таких $n$, что $k(n)<m$ в разложении (24), покажем что тогда утверждение верно для любого числа $n$, в двоичном разложении которого $k(n)=m$. 
Рассмотрим число $n^{\prime}=\sum_{i=0}^{k-1} n_{i+1} 2^{i}$, Тогда

$$
n=2 n^{\prime}+n_{0}
$$

и очевидно справедливо $k\left(n^{\prime}\right)=m-1<m$. Заметим, что при этом для любых $h \in \Omega$ и $i \in \overline{0, m-1}$ выполнено соотношение

$$
h^{\{n /(i+1)\}}=h^{\left\{n^{\prime} / i\right\}} .
$$

Используя следствие 2, находим

$$
\alpha^{[\star n]}=\alpha^{\left[\star 2 n^{\prime}\right]} \star \alpha^{n_{0}}=\left(\sigma^{n^{\prime}}\left(\alpha^{\left[\star n^{\prime}\right]}\right) \cdot \alpha^{\left[\star n^{\prime}\right]}\right) \star \alpha^{n_{0}} .
$$

Выражая $n^{\prime}$ через $n$ из равенства (26), получаем:

$$
\alpha^{[\star n]}=\left(\sigma^{\left\lfloor\frac{n}{2}\right\rfloor}\left(\alpha^{\left[\star n^{\prime}\right]}\right) \cdot \alpha^{\left[\star n^{\prime}\right]}\right) \star \alpha^{n_{0}}=\left(\alpha^{\left[\star n^{\prime}\right]}\right)^{\{n / 1\}} * \alpha^{n_{0}} .
$$

Воспользовавшись предположением индукции, справедливым для числа $n^{\prime}$, получаем:

$$
\alpha^{[\star n]}=\left(\left(\ldots\left(\left(\alpha^{n_{k}}\right)^{\left\{n^{\prime} / k-1\right\}} \star \alpha^{n_{k-1}}\right)^{\left\{n^{\prime} /(k-2)\right\}} \star \ldots \star \alpha^{n_{2}}\right)^{\left\{n^{\prime} / 1\right\}} \star \alpha^{n_{1}}\right)^{\{n / 1\}} \star \alpha^{n_{0}} .
$$

Отсюда и из соотношения (27) следует искомое равенство (25).

Теорема 4. Пусть $(\Omega, \cdot)$ - полугруппа с единищей, $g$ - обратимый элемент. Для локально-медиального группоида $(\Omega, *)$ с операцией $(11)$, где $\sigma, \tau$ удовлетворяют (10), Алгоритм 3 вычисляет степень $g^{[m]}$ со сложностью $O\left(A U T(\sigma, n) \log _{2} n\right)$ onераций в полугруппе $(\Omega, \cdot)$.

Доказательство. Вначале заметим, что на шаге 3.2 происходит изменение элемента $h$ по правилу

$$
h=h^{\{n / i\}} * \alpha^{n_{i-1}} .
$$

Поэтому в результате выполнения шага 3 происходит вычисление правой части выражения (25). По лемме 2 после шага 3 для элемента $h$ выполнено равенство

$$
h=\alpha^{[\star n]} \text {. }
$$

Применяя предложение 7 , получаем

$$
g^{[m]}=g h .
$$

Таким образом, корректность алгоритма доказана.

Оценим трудоёмкость алгоритма. Шаги 1,2 и 4 имеют трудоёмкость $Q_{1}=Q_{2}=$ $Q_{4}=O(1)$ операций в полугруппе $\Omega$.

Трудоёмкость шага 3 оценивается величиной

$$
Q_{3}=\sum_{i=1}^{\left\lfloor\log _{2} n\right\rfloor} A U T\left(\sigma,\left(\left\lfloor\frac{n}{2^{i}}\right\rfloor\right)\right)=O\left(\operatorname{AUT}(\sigma, n) \log _{2} n\right) .
$$

Итоговая трудоёмкость алгоритма - это сумма трудоёмкостей шагов 1-4:

$$
Q=Q_{1}+Q_{2}+Q_{3}+Q_{4}=O\left(A U T(\sigma, n) \log _{2} n\right) .
$$

Тогда трудоёмкость составляет $O\left(A U T(\sigma, n) \log _{2} n\right)$ операций в полугруппе $(\Omega, \cdot)$. 
Замечание 1. Традиционно автоморфизмы задаются действием на образующих элементах. В этом случае трудоёмкость возведения в степень, а значит, и алгоритмов 1-3 оценивается величиной $O\left(\log ^{2}|\Omega|\right)$. Если автоморфизмы $\sigma, \tau$ имеют небольшой по сравнению с мощностью группы порядок, то эта величина становится равной оценке сложности реализации протокола Диффи-Хеллмана.

Сложность возведения в левую степень оценивается аналогично.

\section{2. Первые оценки стойкости предложенных алгорит- MOB}

2.1. Решение задачи дискретного логарифмирования на локально-медиальном группоиде В данном разделе методы дискретного логарифмирования, использующие идеи согласования и сведения к собственным подгруппам, обобщаются на локально-медиальные группоиды. Таким образом, из методов логарифмирования на произвольной абелевой группе остается не обобщенным только $\rho$-метод Полларда.

Модификация алгоритма Гельфонда-Шенкса. Результат предложения 9 позволяет использовать для логарифмирования в локально-медиальном группоиде с операцией (11) идеи алгоритма Гельфонда-Шенкса [6].

\section{Алгоритм 5.}

Дано. $(\Omega, \cdot)$ - полугруппа с единицей, $g, h$ - обратимые элементы полугруппь $(\Omega, \cdot),($ анти-) автоморфизмы $\sigma, \tau \in \widehat{\operatorname{Aut}}(\Omega, \cdot)$.

Выход. Решение х уравнения (2)

$$
g^{[x]}=h .
$$

Шаг 1. Положим $d:=\left\lfloor\sqrt{\operatorname{pot}_{r} g}\right\rfloor+1$.

Шаг 2. Выделим память. Поставим в соответствие элементам группоида ячейки памяти, размером, необходимым для хранения индекса $i \in \overline{1, d}$. Заполним память нулями.

1. Положим $g_{0}=g^{[0]}=e$

2. Вичислим элемент $c=\rho_{d}\left(g, \tau^{-1}(g), \ldots, \tau^{-1}(g)\right)$.

3. Для $i \in \overline{1, d}$ вычислим элементы $g_{i}=g^{[i d]}$, воспользовавиисъ формулой (22):

$$
g_{i}=\sigma^{d-1}\left(g_{i-1}\right) * c,
$$

$u$ по адресу $g_{i}$ запишем в память число $i$.

Шаг 3. Для $j \in \overline{1, d}$ будем вычислять элементы $\beta_{j}=\rho_{j+1}(h, g, \ldots, g)$ и проверять память по адресу $\beta_{j}$.

Если по адресу $\beta_{j}$ записано $i>0$, то выведем ответ $n=i d-j$.

Шаг 4. Если ни для одного из $j$ мы не нашли в памяти ненулевого числа, то въьвдим ответ "Нет решений."

Теорема 6. Алгоритм 5 работает корректно, при этом для реализации алгоритма требуется $O\left(\sqrt{\operatorname{pot}_{r} g}\right)$ операций в полугруппе $(\Omega, \cdot)$ и память обгемом $O\left(\sqrt{\operatorname{pot}_{r} g} \cdot \log _{2}\left(\sqrt{\operatorname{pot}_{r} g}\right)\right)$. 
Доказательство. Доказательство корректности алгоритма состоит из двух частей. Во-первых, покажем, что если уравнение дискретного логарифмирования (2) имеет решение, то для некоторого $j \in \overline{1, d}$ в памяти по адресу $\beta_{j}$ будет ненулевое значение. Для любого натурального $n$ элемент $g^{[n]}$ можно представить в виде $g^{[i d-j]}$, где $j$ меньше $d$. Для этого достаточно поделить $n$ на $d$ с остатком. А значит,

$$
g^{[i d-j]}=h ;
$$

домножив обе части на $g$ по $j$ раз, получаем:

$$
g_{i}=g^{[i d]}=((h * g) * \ldots) * g=\beta_{j} .
$$

Тогда если решение (2) существует, то на шаге 3 для некоторого $j \in \overline{1, d}$ в памяти по адресу $\beta_{j}$ будет ненулевое значение.

Во-вторых, обоснуем корректность выводимого ответа, то есть что из равенства

$$
g_{i}=\beta_{j}
$$

следует, что $(i d-j)$ - решение. Заметим, что так как $g$ - обратимый элемент в $(\Omega, \cdot)$, то уравнение $x * g=f$ для произвольного $f \in \Omega$ имеет единственное решение. А тогда из равенства (28) получаем:

$$
g^{[i d-j]}=h .
$$

На каждом из шагов 2 и 3 выполняется по $O\left(\sqrt{\text { pot }_{r} g}\right)$ операций в полугруппе $(\Omega, \cdot)$. Также на шаге 2 используется память, в которую записывается $\sqrt{\text { pot }_{r} g}$ значений длины $\log _{2}\left(\sqrt{\operatorname{pot}_{r} g}\right)$. Значит, для реализации алгоритма потребуется $O\left(\sqrt{\text { pot }_{r} g}\right)$ операций в полугруппе $\Omega$ и память объемом $O\left(\sqrt{\operatorname{pot}_{r} g} \cdot \log _{2}\left(\sqrt{\operatorname{pot}_{r} g}\right)\right)$.

Обобщение метода сведения к собственным подгруппам. Пусть $(\Omega, \cdot)=(G, \cdot)-$ абелева группа, $(G, *)$ - медиальная квазигруппа с операцией (11). Для решения задачи логарифмирования правых степеней по предложению 7 можем полагать, что автоморфизм $\tau$ - тождественный.

Непосредственной проверкой доказывается следующее предложение, необходимое для описания метода.

Предложение 10. Для произвольного элемента $g \in G$ и для произвольных натуральных $n$ и $k$ выполнено равенство:

$$
\left(g^{[n]}\right)^{k}=\left(g^{k}\right)^{[n]}
$$

Перейдем к описанию метода. Пусть $g, h$ - произвольные элементы квазигруппы $(G, *)$. Решается уравнение вида $g^{[x]}=h$.

1. Рассмотрим первый случай: порядок группы $G$ представим в виде $|G|=p q$, где $p$ и $q$ взаимно простые числа; тогда как следствие нашего уравнения получаем систему

$$
\left\{\begin{array}{l}
\left(g^{[x]}\right)^{p}=h^{p}, \\
\left(g^{[x]}\right)^{q}=h^{q} .
\end{array}\right.
$$

Очевидно, что $\left(g^{[x]}\right)^{p} \in p G$, где $p G=\left\{w^{p} \mid w \in G\right\}-$ подгруппа мощности $|p G|=q$. Автоморфизм $\sigma$ оставляет эту подгруппу на месте. По предложению 10 степень в медиальной квазигруппе перестановочна с групповой степенью. Таким образом, 
если $x$ - решение исходного уравнения, то $x$ - решение уравнения $\left(g^{p}\right)^{[x]}=h^{p}$ в подквазигруппе $(p G, *)$. Потенциал элемента $g^{p}$ в подквазигруппе $(p G, *)$ по предложению 5 будет делить $q$ ord $\sigma$. Аналогичные рассуждения проводятся для подгруппоида $(q G, *)$. А значит, решая уравнения в группоидах $(p G, *)$ и $(q G, *)$, находим все наборы решений вида:

$$
\begin{cases}x \equiv x_{p} & (\bmod (q \operatorname{ord} \sigma)) \\ x \equiv x_{q} & (\bmod (p \operatorname{ord} \sigma))\end{cases}
$$

Данная система имеет решение по модулю $p q \operatorname{ord} \sigma$ тогда и только тогда, когда разрешимо исходное уравнение дискретного логарифмирования.

Таким образом, если исходное уравнение над группоидом $(G, *)$ мощности $|G|=$ $p_{1}^{k_{1}} \cdot \ldots \cdot p_{t}^{k_{t}}$ имеет решение, то его нахождение можно свести к решению уравнений над группоидами примарной мощности.

2. Рассмотрим второй случай: порядок группы $|G|$ имеет вид $|G|=p^{n}$. Пусть экспонента группы $\exp G=p^{k}$. Возведя обе части уравнения в степень $p^{k-1}$, получим уравнение $(2)$ над группоидом $\left(p^{k-1} G, *\right)$. Интересно, что $\left(p^{k-1} G, \cdot\right) \cong \mathbb{Z}_{p} \dot{+} \ldots \dot{+} \mathbb{Z}_{p}$. Таким образом, порядок любого элемента группоида $\left(p^{k-1} G, *\right)$ по предложению 5 делит $p$ ord $\sigma$. Находим все решения $x_{0}$ по модулю $p$ ord $\sigma$.

Решение исходного уравнения представим в виде: $x=x_{0}+x_{1} p$ ord $\sigma$. Тогда исходное уравнение представляется в виде

$$
g^{\left[x_{0}+x_{1} p \operatorname{pot} \sigma\right]}=h,
$$

по следствию 2 данное равенство можно преобразовать к виду

$$
\begin{gathered}
\sigma^{x_{0}}\left(g^{\left[x_{1} p \text { ord } \sigma\right]}\right)=h\left(g^{\left[x_{0}\right]}\right)^{-1}, \\
\left(g^{[\operatorname{ord} \sigma]}\right)^{p x_{1}}=\sigma^{-x_{0}}\left(h\left(g^{\left[x_{0}\right]}\right)^{-1}\right) .
\end{gathered}
$$

Обозначив $g_{1}=\left(g^{[\operatorname{ord} \sigma]}\right)^{p}, h_{1}=\sigma^{-x_{0}}\left(h\left(g^{\left[x_{0}\right]}\right)^{-1}\right)$, получим уравнение

$$
g_{1}^{x_{1}}=h_{1} .
$$

Для решения этого уравнения относительно $x_{1}$ в циклической группе $\left\langle g_{1}\right\rangle$ порядка $p^{k-1}$ можно использовать идеи метода Гельфонда.

Подведём итог. Принципиальное отличие в сложности правого логарифмирования на медиальном группоиде есть лишь на этапе логарифмирования в медиальном группоиде, построенном над группой, изоморфной $\mathbb{Z}_{p} \dot{+} \ldots \dot{+} \mathbb{Z}_{p}$.

Очевидно, что для логарифмирования левых степеней можно привести алгоритмы и методы, аналогичные вышеприведенным, имеющие точно такую же сложность.

\section{2. Верхняя оценка стойкости процедур открытого распределения} ключей Пусть $(\Omega, *)$ - произвольный ПС-группоид. Рассмотрим алгоритм 3 открытого распределения ключей.

Назовём отображение $\delta$ группоида $(\Omega, \star)$ на себя отображением, имитирующим секретный ключ $\left(r, n, a_{1}, \ldots, a_{m}\right)$ абонента $A$, если для любого секретного ключа $\left(s, n, b_{1}, \ldots, b_{n}\right)$ абонента $B$ выполняется равенство

$$
\delta\left(\left[b_{1}\right] \ldots\left[b_{s}\right] g^{\left[b_{s+1}\right] \ldots\left[b_{n}\right]}\right)=\left[a_{1}\right] \ldots\left[a_{r}\right]\left[b_{1}\right] \ldots\left[b_{s}\right] g^{\left[b_{s+1}\right] \ldots\left[b_{n}\right]\left[a_{r+1}\right] \ldots\left[a_{m}\right]},
$$

т. е. если при использовании преобразования $\delta$ вместо преобразования, задаваемого ключом абонента $A$, мы все равно получим истинное значение общего ключа. Кратко будем называть $\delta$ имитирующим отображением. 
Предложение 11. Отображение $\delta$, заданное на $(\Omega, *)$, является имитирующим тогда и только тогда, когда отображение $\delta$ перестановочно с операчиями возведения в правую и левую степенъ и выполняется равенство

$$
\left[a_{1}\right] \ldots\left[a_{r}\right] g^{\left[a_{r+1}\right] \ldots\left[a_{m}\right]}=\delta(g) .
$$

Доказательство. Пусть выполнено условие (30). Тогда для любого секретного ключа абонента В общий ключ, построенный при помощи отображения $\delta$ имеет вид

$$
\delta\left({ }^{\left[b_{1}\right] \ldots\left[b_{s}\right]}(g)^{\left[b_{s+1}\right] \ldots\left[b_{n}\right]}\right)={ }^{\left[b_{1}\right] \ldots\left[b_{s}\right]}(\delta(g))^{\left[b_{s+1}\right] \ldots\left[b_{n}\right]} .
$$

Из (30) получаем:

$$
\left[b_{1}\right] \ldots\left[b_{s}\right](\delta(g))^{\left[b_{s+1}\right] \ldots\left[b_{n}\right]}={ }^{\left[b_{1}\right] \ldots\left[b_{s}\right]}\left(\left[a_{1}\right] \ldots\left[a_{r}\right] g^{\left[a_{r+1}\right] \ldots\left[a_{m}\right]}\right)^{\left[b_{s+1}\right] \ldots\left[b_{n}\right]} .
$$

А это - истинный ключ. Отсюда следует, что $\delta$ - имитирующее отображение.

Обратно, из условий следует равенство (30), так как последнее равносильно (29) при $b_{1}=\ldots=b_{n}=1$. Осталось доказать перестановочность с операциями возведения в степень. При $n=1, s=1$, для любого натурального $b_{1}$ выполнена цепочка тождеств

$$
\delta\left({ }^{\left[b_{1}\right]}(g)\right)={ }^{\left[a_{1}\right] \ldots\left[a_{r}\right]}\left({ }^{\left[b_{1}\right]} g\right)^{\left[a_{r+1}\right] \ldots\left[a_{m}\right]}={ }^{\left[b_{1}\right]}\left(\left[a_{1}\right] \ldots\left[a_{r}\right] g^{\left[a_{r+1}\right] \ldots\left[a_{m}\right]}\right)={ }^{\left[b_{1}\right]}(\delta(g)) .
$$

Аналогично доказывается перестановочность с правыми степенями.

Так как $(\Omega, *)$ - ПС-группоид, то нахождение общего секретного ключа в алгоритме 3 не сложнее решения задачи обобщённого дискретного логарифмирования, т.е нахождения хотя бы одной пары натуральных чисел $u, v$ и набора $\left(x_{1}, \ldots, x_{v}\right)$, $x_{i} \in \mathbb{N}, i \in \overline{1, v}$, удовлетворяющих равенству (5):

$$
\left[x_{1}\right] \ldots\left[x_{u}\right] g^{\left[x_{u+1}\right] \ldots\left[x_{v}\right]}=h,
$$

где $h=\left[a_{1}\right] \ldots\left[a_{r}\right] g^{\left[a_{r+1}\right] \ldots\left[a_{m}\right]}$.

Например, если выполнено соотношение $g^{[r]}=\left[a_{1}\right] \ldots\left[a_{r}\right] g^{\left[a_{r+1}\right] \ldots\left[a_{m}\right]}$, то операция возведения в правую степень $r$ есть имитирующие отображение.

Основываясь на этом, можно предложить следующую атаку.

\section{Алгоритм 7.}

Дано. Группоид $(\Omega, *)$, открытыи ключ абонента $A$ вида $K_{A}=$ $\left[a_{1}\right] \ldots\left[a_{r}\right] g^{\left[a_{r+1}\right] \ldots\left[a_{m}\right]}$.

Выход. Общий секретный ключ $K_{A B}$.

Шаг 1. Решаем уравнение

$$
g^{[x]}=K_{A},
$$

используя алгоритмы, рассмотренные в разделе 2.1 .

Шаг 2. Если решение существует, находим общий ключ $\left({ }^{\left[b_{1}\right] \ldots\left[b_{s}\right]}(g)^{\left[b_{s+1}\right] \ldots\left[b_{n}\right]}\right)^{[x]}$. Иначе переходим $к$ шагу 3.

Шаг 3. Решаем уравнение

$$
{ }^{[x]} g=K_{A},
$$

используя алгоритмы, рассмотренные в разделе 2.1 .

Шаг 4. Если решение существует, то находим общий ключ $[x]\left(\left[b_{1}\right] \ldots\left[b_{s}\right](g)^{\left[b_{s+1}\right] \ldots\left[b_{n}\right]}\right)$. 
Трудоёмкость $Q$ метода определяется сложностью решения уравнений на первом и третьем шагах и составляет $O(L g(\Omega, *))$, где $L g(\Omega, *)$ - трудоёмкость одностороннего логарифмирования. Надежность оценивается снизу величиной

$$
\mathcal{P}=\frac{\left|{ }^{[\mathbb{N}]} g \cup g^{[\mathbb{N}]}\right|}{|\Omega|} .
$$

Таким образом, стойкость (отношение трудоемкости алгоритма к его надежности) оценивается сверху величиной

$$
L g(\Omega, *) \frac{|\Omega|}{\left|{ }^{[\mathbb{N}]} g \cup g^{[\mathbb{N}]}\right|} .
$$

Для локально-медиального группоида удается показать достижимость максимума стойкости алгоритма 2 открытого распределения ключей относительно данной атаки.

Теорема 8. Максимум стойкости Алгоритма 2 открытого распределения ключей относительно алгоритма 7 достигается при $\operatorname{pot}_{r} g \sim \operatorname{pot}_{l} g \sim \sqrt{|\Omega|}, \operatorname{pot}_{l r} g \sim|\Omega| u$ равен $O\left(|\Omega|^{\frac{3}{4}}\right)$.

Доказательство. Трудоёмкость $Q$ метода определяется сложностью решения уравнений на первом и третьем шагах и согласно алгоритму 5

$$
Q=O\left(\sqrt{\text { pot }_{r} g}\right)+O\left(\sqrt{\text { pot }_{l} g}\right)=O\left(\sqrt{\left.\max _{20 t_{r}} g, \operatorname{pot}_{l} g\right\}}\right) .
$$

Таким образом, для некоторой константы $a \in \mathbb{R}$

$$
Q=a \sqrt{\max \left\{\operatorname{pot}_{r} g, \operatorname{pot}_{l} g\right\}} .
$$

Очевидно, выполняются неравенства

$$
\operatorname{pot}_{l r} g \leqslant \operatorname{pot}_{l} g \cdot \operatorname{pot}_{r} g ; \quad \operatorname{pot}_{l r} g \leqslant|\Omega| \text {. }
$$

Вероятность $\mathcal{P}$ нахождения истинного общего ключа с помощью предложенного алгоритма есть вероятность разрешимости уравнений на первом или третьем шагах:

$$
\mathcal{P}=\frac{\operatorname{pot}_{r} g}{\operatorname{pot}_{l r} g}+\frac{\operatorname{pot}_{l} g}{\operatorname{pot}_{l r} g} \geqslant \frac{\max \left\{\operatorname{pot}_{r} g, \operatorname{pot}_{l} g\right\}}{\operatorname{pot}_{l r} g} .
$$

Тогда, пользуясь (31), стойкость процедуры относительно данного метода можно оценить следующим образом:

$$
\frac{Q}{\mathcal{P}} \leqslant \frac{a \sqrt{\max \left\{\operatorname{pot}_{r} g, \operatorname{pot}_{l} g\right\}}}{\max \left\{\operatorname{pot}_{r} g, \operatorname{pot}_{l} g\right\}} \operatorname{pot}_{l r} g=\frac{a \operatorname{pot}_{l r} g}{\sqrt{\max \left\{\operatorname{pot}_{r} g, \operatorname{pot}_{l} g\right\}}} .
$$

При потенциалах элемента $g$, близких к $|\Omega|$, имеем соотношение

$$
\frac{Q}{\mathcal{P}}=O(\sqrt{|\Omega|})
$$

а это - оценка сложности логарифмирования в "классическом" случае.

Найдём максимум величины $\frac{Q}{\mathcal{P}}$. Имеем соотношение

$$
\frac{Q}{\mathcal{P}}=\frac{a \operatorname{pot}_{l r} g}{\sqrt{\max \left\{\operatorname{pot}_{r} g, \operatorname{pot}_{l} g\right\}}} \leqslant \frac{\operatorname{pot}_{l r} g}{\sqrt{\left(\operatorname{pot}_{r} g+\operatorname{pot}_{l} g\right) / 2}} .
$$


Используя неравенство между средним арифметическим и средним геометрическим, получаем:

$$
\frac{Q}{\mathcal{P}} \leqslant \frac{a \operatorname{pot}_{l r} g}{\sqrt{\sqrt{\left(\operatorname{pot}_{r} g \cdot \operatorname{pot}_{l} g\right)}}}=\frac{a \operatorname{pot}_{l r} g}{\left(\operatorname{pot}_{r} g \cdot \operatorname{pot}_{l} g\right)^{1 / 4}} .
$$

Отсюда следуют соотношения:

$$
\frac{Q}{\mathcal{P}} \leqslant\left\{\begin{aligned}
\frac{a|G|}{|\Omega|^{1 / 4}} & \leqslant a|\Omega|^{3 / 4}, \text { если }\left(\operatorname{pot}_{r} g \cdot \operatorname{pot}_{l} g\right) \geqslant|\Omega|, \\
a\left(\operatorname{pot}_{r} g \cdot \operatorname{pot}_{l} g\right)^{3 / 4} & \leqslant a|\Omega|^{3 / 4}, \text { если }\left(\operatorname{pot}_{r} g \cdot \operatorname{pot}_{l} g\right) \leqslant|\Omega| .
\end{aligned}\right.
$$

Очевидно, что оценка $\frac{Q}{\mathcal{P}} \leqslant a|\Omega|^{3 / 4}$ достижима при $\operatorname{pot}_{r} g \sim \operatorname{pot}_{l} g \sim \sqrt{|\Omega|}$ и $\operatorname{pot}_{l r} g \sim|G|$.

Группоиды, в которых оценка достижима, существуют.

Пример 2. Пусть $\langle a\rangle$ - циклическая группа порядка $m$, и $(\Omega, \cdot)=\langle a\rangle \otimes\langle a\rangle-$ внешнее прямое произведение групп. Тогда группоид $(\Omega, *)$, определяемый равенством (11) при $\tau=\operatorname{id}_{\Omega},-$ тождественный автоморфизм, и $\sigma(x, y)=(y, x)$, - медиальная квазигруппа.

Рассмотрим элемент $g=(a, e) \in \Omega$. Нетрудно показать, что $\operatorname{pot}_{r} g=m, \operatorname{pot}_{l} g=$ $2 m$ и $\operatorname{pot}_{l r} g>\phi(m) m$, где $\phi(m)-$ функция Эйлера.

Наряду с возведением в степень в локально-медиальном группоиде в качестве имитирующего гомоморфизма может быть использована операция возведения в степень в исходной полугруппе. Такая атака успешна в случае, когда, например, каждый из автоморфизмов (10), задающих операцию в группоиде, есть возведение в степень.

\section{3. Медиальная квазигруппа на группе точек эллип- тической кривой}

Пусть $p>2$ - простое число, $q=p^{k}, F_{q}$ - поле Галуа и $A, B \in F_{q}$. Эллиптическая кривая $E=E\left(F_{q}\right)$ есть множество решений $P=(a, b) \in F_{q}^{2}$ уравнения

$$
y^{2}=x^{3}+A x+B
$$

в совокупности с бесконечно удаленной точкой $\Theta$. В соответствии с [11] на $E\left(F_{q}\right)$ определяется структура абелевой группы $(E, \oplus)$ с нейтральным элементом $\Theta$. Сложность реализации операции $\oplus$ оценивается величиной $O(1)$ операций в поле $F_{q}$.

В общем случае трудоёмкость задачи дискретного логарифмирования на группе точек эллиптической кривой имеет корневую оценку. В связи с этим, медиальные квазигруппы, построенные на них, могут оказаться подходящими алгебраическими структурами для построения алгоритма открытого распределения ключа с большей стойкостью.

При условии $A, B \in F_{p}$ существует автоморфизм $\sigma$ группы $(E, \oplus)$, определяемый равенством

$$
\sigma((x, y))=\left(x^{p}, y^{p}\right) .
$$

Пусть $\tau$ - тождественный автоморфизм этой группы. Зададим на $E$ операцию * равенством $(11)$, тогда $(E, *)$ - медиальная квазигруппа. 
Оценим стойкость алгоритма 2 открытого распределения ключей, реализованного на ПС-группоиде $(E, *)$.

Очевидно, что построение имитирующего отображения в случае, когда $\sigma(P)=$ $k P$, равносильно логарифмированию на группе точек эллиптической кривой, а значит, с точки зрения анализа интерес представляет случай, когда автоморфизм Фробениуса $\sigma$ на кривой $E\left(F_{q}\right)$ не соответствует умножению точки ни на какое целое число. В дальнейшем будем рассматривать только такие кривые.

Группа эллиптической кривой $E\left(F_{q}\right)$ порядка $m$ изоморфна группе $\mathbb{Z}_{m_{1}} \dot{+} \mathbb{Z}_{m_{2}}$, где $m=m_{1} m_{2}, m_{2} \mid\left(m_{1}, q-1\right)$.

Если $m_{2}=1$, то $(E, \oplus)$ - циклическая группа. В этом случае стойкость общего секретного ключа из алгоритмов 1-3 не превосходит трудоёмкости дискретного логарифмирования на кривой $\left(E\left(F_{q}\right), \oplus\right)$.

Рассмотрим интересный частный случай.

Пример 3. Пусть эллиптическая кривая $E\left(F_{p^{2}}\right)$, заданая уравнением $(32)$ при $A, B \in F_{p}$, изоморфна группе $\mathbb{Z}_{n} \dot{+} \mathbb{Z}_{n}, n \in\{p-1, p+1\}$. Такие кривые относятся к классу суперсингулярных [11]. Тогда автоморфизм $\sigma$, задаваемый равенством $(33)$, имеет порядок 2 , и можно показать, что медиальная квазигруппа $(E, *)$ содержит такой элемент $g$, что $\operatorname{pot}_{l} g=n, \operatorname{pot}_{r} g=2 n, \operatorname{pot}_{l r} g \geqslant \varphi(n) n$, где $\varphi-$ функция Эйлера.

Таким образом, стойкость алгоритма 2 относительно алгоритма 7 в данном примере будет достигать своего максимума $O\left(n^{3 / 2}\right)$.

Замечание 2. Порядки точек эллиптической кривой из предыдущего примера не превосходят $n$. Таким образом, стойкость процедуры Диффи-Хеллмана, построенной на $(E, \oplus)$, оценивается сверху величиной $n^{1 / 2}$.

Однако для медиальных группоидов, построенных на группе точек эллиптической кривой, можно предложить другие атаки, основанные на построении имитирующего отображения.

Атака 1. Любая комбинация правых и левых степеней элемента $g$ такого группоида представляется в виде

$$
{ }^{[l]} P^{[r]}=k_{1} P \oplus k_{2} \sigma(P)
$$

для некоторых натуральных $k_{1}, k_{2}$.

Гомоморфизм $\delta$, действующий на элементах группы $E$ по правилу

$$
\delta(Q)=k_{1} Q \oplus k_{2} \sigma(Q)
$$

очевидно перестановочен с автоморфизмом $\sigma$ и тождественным $\tau$. А значит, $\delta$ будет имитирующим гомоморфизмом.

Таким образом, задача нахождения общего секретного ключа сводится к задаче построения представления элемента $K_{A}$ в виде $(34)$ в подгруппе абелевой группы $E$, порожденной элементами $P$ и $\sigma(P)$.

Данная задача является частным случаем задачи двумерного дискретного логарифмирования (см., например, [8]), которая в случае группы точек эллиптической кривой формулируется как нахождение коэффициентов $k_{1}, k_{2}$, удовлетворяющих равенству

$$
Q=\left[k_{1}\right] P \oplus\left[k_{2}\right] S
$$

для некоторых фиксированных точек $P, S, Q \in E$. 
Для решения данной задачи в работе [9] предложен алгоритм, использующий идеи метода согласования. Алгоритм требует $O(\sqrt{|E|})$ операций в поле $F_{q^{m}}$ и $O(\sqrt{|E|})$ ячеек памяти.

Позднее, в работе [8] Gaudry и Schost предложили вероятностный алгоритм, основанный на парадоксе дней рождений, его трудоемкость оценивается величиной $O(\sqrt{|E|})$.

Для описания следующей атаки введём определения.

Пусть $m=\left|E\left(F_{q}\right)\right|$ и $(m, p)=e$. Обозначим $E\left(\overline{F_{q}}\right)$ кривую, заданную уравнением $(32)$, над алгебраическим замыканием поля $\overline{F_{q}}$. Тогда каждая точка кривой $E\left(F_{q}\right)$ является одновременно точкой кривой $E\left(\overline{F_{q}}\right)$, так как поле $\overline{F_{q}}$ содержит поле $F_{q}$.

Положим $E[m]=\left\{P \in E\left(\overline{F_{q}}\right):[m] P=\Theta\right\}$. В [11] показано, что $E[m]$ является подгруппой группы точек эллиптической кривой $E\left(F_{q^{t}}\right)$ для некоторого натурального $t$.

Очевидно, справедливы неравенства $E\left(F_{q}\right)<E[m]<E\left(F_{q^{t}}\right)$.

Пусть $M_{m}=\left\{\mu \in F_{q^{t}}: \mu^{m}=1\right\}-$ множество корней из 1 порядка $m$ поля $F_{q^{t}}$. В монографии [11] вводится билинейное отображение $e_{m}: E[m] \times E[m] \rightarrow M_{m}$, называемое спариванием Вейля. Показывается, что существует такая точка $T \in E\left(F_{q}\right)$, что отображение $\psi: E[m] \rightarrow F_{q^{t}}$, задаваемое по правилу $\psi(S)=e_{m}(S, T)$, есть изоморфное вложение. Параметр $t$ этого отображения называют $M O V$ (Menezes-Okamoto-Vanstone)-cmепенъю группы $E\left(F_{q}\right)$.

Замечание 3. Эффективные алгоритмы вычисления значения спаривания Вейля предложены в [10].

Если MOV-степень $t$ рассматриваемой эллиптической кривой невелика, то можно предложить следующую атаку.

Докажем вспомогательное утверждение.

Предложение 12. Для любой такой точки $P$ порядка $m$, что $\sigma(P) \neq k P, \forall k \in \mathbb{N}$, группы Е выполнено:

$$
e_{m}(P, \sigma(P)) \neq 1 \text {. }
$$

Доказательство. Так как спаривание Вейля не вырождено, то существуют такие точки $S, T \in E$ порядка $m$, что $e_{m}(S, T) \neq 1$. Точки $S, T$ представляются в виде $S=t_{1} P \oplus t_{2} \sigma(P), T=t_{3} P \oplus t_{4} \sigma(P)$. Следовательно,

$$
e_{m}(S, T)=e_{m}(P, \sigma(P))^{t_{1} t_{4}-t_{2} t_{3}} \neq 1 \text {. }
$$

А значит, $e_{m}(P, \sigma(P)) \neq 1$.

Атака 2. Будем искать имитирующий гомоморфизм $\delta$ вида (34).

Для нахождения $k_{1}, k_{2}$ рассмотрим следующие спаривания:

$$
\begin{gathered}
e_{m}\left({ }^{[l]} P^{[r]}, \sigma P\right)=e_{m}\left(k_{1} P+k_{2} \sigma(P), \sigma(P)\right)=e_{m}(P, \sigma(P))^{k_{1}}, \\
e_{m}\left({ }^{[l]} P^{[r]}, P\right)=e_{m}\left(k_{1} P+k_{2} \sigma(P), P\right)=e_{m}(\sigma(P), P)^{k_{2}} .
\end{gathered}
$$

Получаем два логарифмических уравнения над полем $G F\left(q^{2 t}\right)$

$$
\begin{gathered}
e_{m}(P, \sigma(P))^{k_{1}}=e_{m}\left({ }^{[l]} P^{[r]}, \sigma(P)\right), \\
e_{m}(\sigma(P), P)^{k_{2}}=e_{m}\left({ }^{[l]} P^{[r]}, P\right) .
\end{gathered}
$$


Таким образом, мы получаем информацию о коэффициентах $k_{1}, k_{2}$. Трудоёмкость атаки равна сложности двух логарифмирований в поле $F_{q^{t}}$.

Если исходная кривая суперсингулярная, то трудоёмкость атаки не превосходит сложности двух логарифмирований в поле $F_{q^{2}}$.

Остается отметить, что трудоёмкость построения суперсингулярных кривых с требуемыми свойствами требует минимальных затрат на предварительном этапе, в то время как построение кривых, использующихся в российских стандартах цифровой подписи (ГОСТ 34.10), - это трудоёмкий процесс, занимающий длительное время.

\section{Список литературы}

1. Словарь криптографических терминов, под ред. Погорелова Б. А. и Сачкова В.Н., МЦНМО, Москва, 2006, 94 с.

2. Алфёров А. П., Зубов А. Ю., Кузьмин А. С., Черемушкин А. В., Основы криптографии, Гелиос АРВ, Москва, 2005, 480 с.

3. Белявская Г. Б., Табаров А. Х., “Тождества с подстановками, приводящие к линейности квазигрупп", Дискретная математика, 21:1 (2009), 36-51.

4. Василенко О.Н., Теоретико-числовые алгоритмы в криптографии, 2-е изд., МЦНМО, Москва, 2007, 328 с.

5. Глухов М. М., Елизаров В. П., Нечаев А. А., Алгебра, т. 2, Гелиос АРВ, Москва, 2003, $414 \mathrm{c}$.

6. Глухов М.М., Круглов И.А., Пичкур А.Б., Черемушкин А.В., Введение в теоретико-числовые методы криптографии, учебное пособие, Лань, Санкт-Петербург, 2010, $400 \mathrm{c}$.

7. Diffie W., Hellman M. E., "New directories in cryptography", IEEE Trans. Inf. Theory, 1976, 29-40.

8. Gaudry P. and Schost E., "TA low-memory parallel version of Matsuo, Chao and Tsujii's algorithm": in D. A. Buell, editor, Proceedings of Algorithm Number Theory Symposium ANTS VI, volume 3076 of LNCS, Springer-Verlag, 2004, 200-222.

9. Matsuo K., Chao J., and Tsujii S., "An improved baby step giant step algorithm for point counting of hyperelliptic curves over Finite Felds": In C. Fiecker and D. Kohel, editors, Proceedings of Algorithm Number Theory Symposium - ANTS V, volume 2369 of LNCS, Springer-Verlag, 2004, 461-474.

10. Menezes A., P. van Oorschot, Vanstone S., "Reducing elliptic curve logarithms to logarithms in a finite field", IEEE Trans. Inf. Theory, 1993, 1639-1646.

11. Silverman J., The Arithmetic of the Elliptic Curves, Springer, 1986, 513 pp. 